\title{
Complementary and alternative medicine
}

\author{
Sir Richard Thompson
}

Complementary and alternative medicine (CAM) is a group of diverse medical and healthcare systems, practices and products that are not presently considered to be part of conventional medicine. Complementary and alternative medicine covers a wide range of therapies ranging from the well-known acupuncture, homoeopathy and osteopathy, through herbal medicines to reflexology and prayer. Sometimes they are in addition to medical treatment (complementary), sometimes instead of it (alternative), and sometimes simply as a pleasant experience, such as aromatherapy. These treatments, within the NHS or paid for by the patient, are clearly popular with patients judging by their extensive use. Of the UK population $10 \%$ use a CAM treatment each year, while half the population, frequently younger patients, have used CAM therapy at some time. In the USA, patients spend $\$ 17$ billion on CAM per annum. ${ }^{1,2}$ Many patients believe strongly that these treatments are beneficial, improving their well-being and lifestyle, but over half of UK doctors feel that they should not be funded by the NHS. Are these positive reports simply chance, as part of the variable natural histories of disease, or are they real?

In 1999, the House of Lords Select Committee on Science and Technology set up a subcommittee, chaired by John Walton, to examine the place of CAM in modern medicine. The subcommittee concluded that the evidence base was poor, more regulation was needed, and more research was essential. ${ }^{3}$ In 1991, the Royal College of Physicians ran a successful conference entitled 'The science behind $\mathrm{CAM}^{4}$ and sponsored a second in 2001 with the US National Center for Complementary and Alternative Medicine (Bethesda, MD) entitled 'Can alternative medicine be integrated into mainstream care?. ${ }^{5}$ It was against this background that the College's Subcommittee on CAM ${ }^{6}$ now re-named the Integrated Health Committee, arranged a conference entitled 'The science and art of healing: understanding the therapeutic response' to look at the general process of care behind CAM treatments. The latest conference brought together patients, CAM practitioners and researchers, psychologists and sceptics. It did not examine the evidence behind CAM. Champions of integrative diagnosis and treatment, including patients, urge physicians to consider the physiology of the whole patient, body and mind, and not just the conventional system that exhibits the current symptoms. The Prince of Wales Foundation for Integrated Medicine (now the Prince's Foundation for Integrated Health) was founded in 1976 to promote the use of, and training in, this type of approach. ${ }^{7}$ Indeed, many primary care practices now provide some of the better recognised therapies either on the NHS, if the local primary care trusts will fund the service, or privately, and their practitioners are enthusiastic. They point out that CAM treatments are cheaper than many drug treatments, prevent referrals to, for instance, orthopaedic clinics, and are generally safer than orthodox treatments. Such an approach can unlock difficult diagnostic situations and lead to improvement or cure. Nevertheless, it is worth remembering that cervical manipulation ${ }^{8}$ and herbal treatments, ${ }^{9,10}$ for example, are not always safe, while poor diagnosis can badly delay referral, diagnosis and treatment of underlying serious conditions.

Few would argue that CAM therapy is effective in serious life-threatening conditions, such as type I diabetes mellitus or gastrointestinal bleeding, but physicians know that there are many other patients who do not respond to their treatment, or have symptoms which we do not know how to treat, depending upon your point of view. Think of chronic fatigue syndrome for example. Nevertheless each specialty has such patients, for example headaches in neurology, irritable bowel in gastroenterology, chest pain in cardiology and fibromyalgia (chronic widespread pain) in rheumatology. It may be easy to exclude a serious underlying pathology by examination and investigation, concluding in frustration that there is nothing wrong and hence, by implication, that the pain is imaginary. The conference showed that brain imaging can now detect abnormal activity in the brain in patients complaining, for instance, of fibromyalgia, or in burning mouth syndrome, ${ }^{11}$ and so it seems likely that such pain can be real. Several conditions with ill-defined pathology apparently may follow one another or come together in the same patient, suggesting that there is a generalised underlying neurological or psychological pattern.

Many doctors, brought up as they are in a scientific environment and understandably convinced by the power of double blind controlled trials, find it difficult or impossible to accept the efficacy of many CAM treatments. ${ }^{12}$ It would be reassuring to have

\section{Sir Richard} Thompson KCVO DM FRCP, Treasurer, Royal College of Physicians, London

This conference was held at the Royal College of Physicians on 13 September 2007 and was organised jointly with the Royal College of General Practitioners and The Prince's Foundation for Integrated Health 
scientific and plausible explanations of the mechanism of a CAM treatment or, even better, an animal model of the disease and treatment. This may not, however, be essential and was not available for orthodox treatments until recently; indeed, the evidence base for many orthodox treatments remains poor. It is said that the majority of orthodox treatments given by primary care doctors are not evidence based. Research may be able to find unexpected scientific explanations for CAM treatments, for all are open to a quantitative trial - it may not be as easy as a drug trial, but studies are feasible, sometimes with elaborate arrangements to provide complete sham treatments. Unfortunately the main funding bodies are reluctant to support such studies as they seem less rigorous than laboratory-based experiments. The Department of Health established CAM PhD fellowships in 2002, and in the USA there has been a National Center (initially an Office) for CAM within the National Institutes of Health (Bethesda, MD) since 1992.

It is increasingly recognised that the so-called placebo effect may be an important part of many orthodox and CAM treatments. ${ }^{13}$ Clearly not with insulin treatment, but there is much evidence, for instance, that drugs used for mild depression are

\section{Conference programme}

What patients want from doctors

Pat Lister, Patient

I The patient's story

Dr Charlotte Paterson, Senior Research Fellow, Peninsula

Medical School, Universities of Exeter and Plymouth

How patients perceive complementary medicine

Dr Felicity Bishop, Research Fellow, University of Southampton

\section{LANGDON-BROWN LECTURE}

I The placebo effect and the power of belief

Professor Irving Kirsch, Chair in Psychology, University of Hull

I The nature of success with placebo treatment Professor Robert Allan, Director of Publications and Editor, Clinical Medicine, Royal College of Physicians

- How can we define specific and non-specific within a complex system?

Professor Michael Hyland, Professor of Health Psychology, University of Plymouth

I Efficacy versus effectiveness, what really matters? Professor David Price, GPs in Airways Group Professor of Primary Care Respiratory Medicine, University of Aberdeen

\section{Imaging expectancy for pain}

Professor Anthony Jones, Professor of Neuro-Rheumatology, Manchester University

- Can we image a specific effect for acupuncture? Dr Peter White, Postdoctoral Research Fellow and Lecturer in Physiotherapy, School of Health Professions and Rehabilitation Sciences, University of Southampton

- Delivering integrated care

Panel discussion on delivering integrated care only slightly better than placebo drug. This slight superiority in trials may only be due to the patients' side effects, which causes them to feel that they are receiving the active treatment and to respond psychologically; when there are no side effects the subject not unnaturally believes it to be the placebo. Furthermore, it is easy to become cynical when thinking of type 1 and 2 errors in trials, of the publication bias against negative trials, or of the influence of pharmaceutical companies. We were informed that there is clear evidence of this when reviewing the overall results of unpublished drug trials in the database of the Federal Drug Administration.

Controlled trials of CAM treatments are regularly published in standard medical journals, where they usually arouse heated debate in the correspondence section. Many conclude from the inconsistency of the results of the trials and meta-analyses that CAM treatments have at best a small, clinically insignificant intrinsic effect. But that is not the whole story, for the most rigorous trials have attempted to compare the CAM procedure against controls, and have, therefore, left out an important part of the whole treatment, namely the warm, empathetic and, compared to the NHS, long consultation. Patients voice their wish to have a longer, more discursive consultation with their primary or secondary care physician, with a full discussion of their mental and physical problems, lifestyle and life history. This, in one sense, may be a placebo treatment, but is nevertheless a valuable one, that underlies the efficacy of CAM treatments, while explaining the frequent failure to prove their efficacy in carefully designed trials. ${ }^{14}$

All physicians, like their predecessor witch doctors, rely on such a placebo effect. The confidence with which a diagnosis is given and treatment prescribed is an important factor in its success. There is evidence that the colour of medicines influences their efficacy, while even the insertion of a cardiac pacemaker carries a powerful placebo effect. ${ }^{15}$ Should orthodox doctors therefore prescribe their placebos in the form of coloured medicines and accompany this with strong reassurance, oozing confidence of their efficacy? At the conference some patients argued that this would undermine trust and treat them as children. But if the placebo is fully explained to the patient, then surely its well documented efficacy would often be lost? Should the homoeopath explain that succussed water is a placebo and thereby probably negate its efficacy? Should the acupuncturist explain that it is the empathetic consultation that is effective and not the needles? We need more research into the power of the consultation alone in the setting of both orthodox and CAM treatments.

Is it therefore the process of care rather than any specific pharmaceutical treatment that can often be effective? Patients universally want more time with their doctors, while the latter are trained not to listen. ${ }^{16}$ Perhaps, after all, CAM practitioners are simply telling orthodox physicians that longer, but not too long, and fuller consultations and advice on lifestyles should be taught in medical schools and practised in the NHS? Would this then decrease the popularity of CAM treatments? Something tells me that this approach would require more doctors, and hence allow the employment of the increasing number of UK and international physicians. 


\section{References}

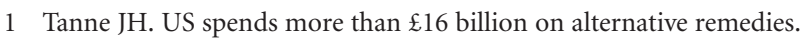
BMJ 1998;317:1408.

2 MacLennan AH, Wilson DH, Taylor AW. Prevalence and cost of alternative medicine in Australia. Lancet 1996;347:569-73.

3 House of Lords Select Committee on Science and Technology. Complementary and alternative medicine, sixth report. London: House of Lords, November 2000.

4 Holgate ST. Does homoeopathy work and if so how? In: Meade T (ed), Science-based complementary medicine. London: Royal College of Physicians, 1998;7-21.

5 Brien S, Lewith GT. Why are you doctors? The importance of care and compassion. Clin Med 2001;1:223-6.

6 Lewith GT, Breen A, Filshie J et al. Complementary medicine: evidence base, competence to practice and regulation. Clin Med 2003; 3:235-40.

7 The Prince's Foundation for Integrated Health. www.fih.org.uk/index.html

8 Ernst E. Adverse effects of spinal manipulation: a systematic review. J Roy Soc Med 2007;100:1-9.
9 Finall AI, McIntosh SA, Thompson WD. Subcutaneous inflammation mimicking metastatic malignancy induced by injection of mistletoe extract. BMJ 2006;333:1293-4.

10 Henley DV, Lipson N, Korach KS, Bloch A. Prepubertal gynecomastia linked to lavender and tea tree oils. N Engl J Med 2007;356:479-85.

11 Fedele S, Fricchione G, Porter SR, Mignogna MD. Burning mouth syndrome (stomatodynia). QJ Med 2007;100:527-30.

12 Kerr D. In search of truth. J Roy Coll Phys Lond 1996;30:405.

13 Pearce JMS. The placebo enigma. QJ Med 1995;88:215-20.

14 Wigley R. When is a placebo effect not an effect? Clin Med 2007;7: 450-2.

15 Sud S, Massel D, Klein GJ et al. The expectation effect and cardiac pacing for refactory vasovagal syncope. Am J Med 2007;120:54-62.

16 Launer J. The art of not listening. QJ Med 2007;100:537-8. 